\begin{tabular}{|c|c|c|}
\hline & PORT SAID ENGINEERING RESEARCH JOURNAL \\
Faculty of Engineering - Port Said University
\end{tabular}

\title{
Joint Resistance of Bolted Power Connectors as Influenced by Overlap Area and Tightening Torque of Contact Parts
}

\author{
Ghareeb Moustafa \\ Suez Canal University, Egypt. \\ ghareeb_moustafa@eng.suez.edu.eg
}

\begin{abstract}
:
Electrical contacts consist of two parts where the surfaces are in contact and where the actual physical contact occur just in a few contact asperity points scattered over the whole apparent contact area. the effect of material resistance on the initial joint resistance of bolted power bus-bar have been investigated experimentally by measuring the current distribution along the over lapping region of bolted joint, moreover the effect of increasing torque on the current distribution along contact area are discussed, in addition, a computer simulation based upon the finite element method was introduced to calculate the ideal joint resistance and compared with that obtained experimentally. Furthermore, the effect of increasing bolted size (hole diameter) on the initial joint resistance was calculated. The study shows that the current distribution is not homogenous along the contact interface and increasing tightening torque has no effect on the current distribution along the contact area.
\end{abstract}

Keywords: Connectors, Initial Resistance, current distribution, Ideal joint.

\section{Introduction}

In a power plant, electrical connectors are tremendous. They exist in the high-voltage systems to provide the electrical interface between the power plant and the transmission system. There are large numbers of electrical connectors found in high voltage Substations, medium - and low-voltage switchgear, segregated and non-segregated bus, equipment and structure grounding systems, control cabinets, and other electrical enclosures. There are also a large number of connectors in electronic and computer equipment, many of which were installed at the factory contact mechanics of electrical contacts has been extensively investigated through the last decades by many researchers $[1,2]$

Elements and devices of the power transmission and distribution systems are connected by a large number of different electrical joints which are subjected to ageing processes. The electrical resistance of joints will increase during this ageing time. Because of this, the joint temperature caused by increased power losses can be so high that the joints themselves or the associated insulation material will be destroyed. Thus, the reliability of the electrical power supply system will be determined significantly by the high current joints, it is well known that the electrical joint resistance of clean contacts depends on such parameters as: normal force, contact micro hardness, electrical resistivity and surface texture (roughness). Traditionally, surface texture parameters are defined by the variance of the height and the slope of the surface $[4,5]$.

It is well established that the electrical contact resistance of clean contacts, depends on such parameters as: normal force, contact micro hardness, electrical resistivity and surface texture (roughness) traditionally, surface texture parameters are defined by the variance of the height and the slope of the surface [6]. The most significant quantity to assess is the joint resistance to guarantee reliable operation of the joint. The joint resistance should be no greater than limiting value. Such limiting values do not exist at present. Furthermore, it is known that the life time of an electrical joint will be determined significantly by the joint resistance $\mathrm{R}_{0}$ after assembly (before aging) [7]. The quickness of ageing depends on the performance factor $\mathrm{k}$ which equal the joint resistance divided by the resistance of an unjointed bar of the same length, it has proven that maximum initial performance factor must be equal or less than 1.5 to have a life time $25 \ldots 30$ years for bolted joint $[8,9]$. There is no article found discussed the effect of material resistance on the initial joint resistance. 
The aim of the work presented here is to bring a contribution to the factors effect on initial joint resistance in bolted power connection. Indeed, it was interesting to study the current distribution over the contact area based on the voltage measurement over the connection region. Furthermore, a rigid model of finite element analysis was established in this work, the effect of increasing hole diameter on the joint resistance of bolted copper bus-bar without contact surface was investigated. Moreover, the influence of increasing overlap distance without contact surface was investigated and compare with that obtained experimentally in [1].

\section{Load bearing area}

The load bearing area Figure 1 can be categorized into three parts: metallic contact areas, quasi-metallic spots, and insulating film areas. The short name a-spot for the conducting contact areas, referring to the radius "a" of a circular contact area, is a widely accepted term. To sum up, not only is the load bearing contact area very small, but also that only a fraction of it may be electrically conducting.

In a bulk electrical contact interface, the electrical current lines get distorted. The flow lines bundle together to pass through a-spots. Thus, less volume of material is now available for current conduction, causing increase in electrical resistance. This increase in resistance is defined as "constriction Resistance, Holm has shown that the constriction resistance for a single aspot can be expressed as [1]:
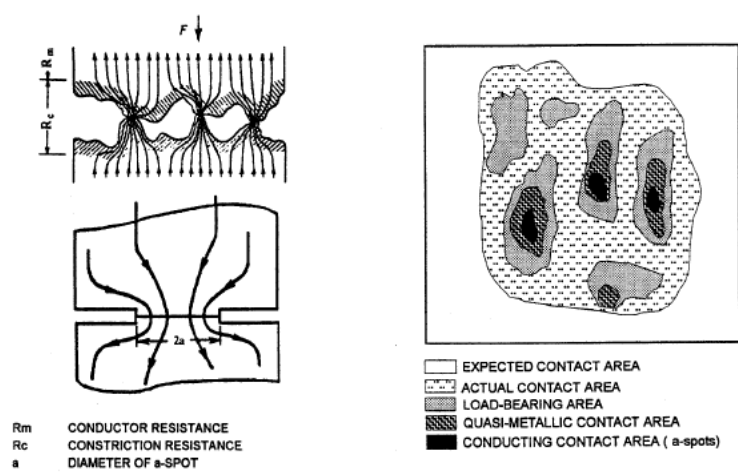

Figure 1: Schematic of load bearing area and current concentration [10].

$$
R_{g}=\left(\mathrm{p}_{1}+\mathrm{p}_{2}\right) / 4 \mathrm{a}
$$

Where $\rho 1$ and $\rho 2$ are resistivity's of the contacting metals, and $\mathrm{a}$ is the radius of the metal-to-metal contact area (contact spot). If the two contacting metals are the same, then the constriction resistance becomes

$$
\mathrm{R}_{\mathrm{s}}={ }^{\mathrm{P}} / 2 \mathrm{a}
$$

Because the metals are not clean, the passage of electric current may be affected by thin oxide, sulphide, and other inorganic films usually present on metal surfaces. In the presence of any contaminating film with relatively high electrical resistivity, total contact resistance RC increases further. This additional is called as "Film Resistance Rf ". Thus, total interfacial resistance is the sum of constriction and film resistance Figure 11

$$
\begin{array}{ll}
\mathrm{R}_{\mathrm{c}}=\mathrm{R}_{\mathrm{s}}+\mathrm{R}_{\mathrm{f}} & \text { Eq. } 3 \\
\mathrm{R}_{\mathrm{f}}={ }^{\sigma} / \mathrm{\pi a} & \text { Eq. } 4
\end{array}
$$

Where $\sigma$ is the resistance per area of the film. In most practical applications, the contribution of these films to the total contact resistance is of minor importance because the contact spots are usually created by the mechanical rupture of surface films.

When the current is confined to flow through the conducting spots (a-spots), the temperature of the contact point $\left(\mathrm{T}_{\mathrm{c}}\right)$ may be higher than that of the bulk temperature $\left(\mathrm{T}_{\mathrm{b}}\right)$. Hence, the increase in constriction resistance over the resistance that would exist if the metal-metal contact were continuous across the entire contact area can be expressed as [2]:

$R_{c}=R_{c}(0)\left[1+2 / 3 \alpha\left(T_{c}-T_{b}\right)\right]$

Eq. 5

The term $\left(T_{c}-T_{b}\right)$ is called the super temperature and is related to the voltage drop across the contact interface (V) as follows

$\mathrm{T}_{\mathrm{c}}^{2}-\mathrm{T}_{\mathrm{b}}^{2}=\mathrm{V}^{2} / 4 \mathrm{~L}$

Eq. 6

Where $\mathrm{L}$ is the Wiedemann-Franz Lorenz number with the value of $2.45 \times 10^{-8}(\mathrm{~V} / \mathrm{K})^{2}$ It is clear that even a relatively small increase in the contact voltage drop (V) can raise the super temperature considerably, enough to produce basic metallurgical changes such as softening or even melting of the conducting areas.

\section{Experimental Data}

\subsection{Types of connectors}

The tests applied on bolted connectors were performed using copper bus- bars (Figure 2 ) $40 \times 10 \mathrm{~mm}^{2}$. Three joints were used namely:

1- Ideal copper joint without contact interface and with $40 \mathrm{~mm}$ overlap (this joint are made from $40 \mathrm{x}$ $20 \mathrm{~mm}^{2}$ copper bus- bar, which machined to be like normal joint but without contact surface)

2- Bolted copper joint with 40 mm overlap

3- Bolted copper joint with $80 \mathrm{~mm}$ overlap

\subsection{Circuits diagrams}

The contact surface was assembled to form the connector and the DC joint resistance was measured using micro- 
ohmmeter. The resistance was measured on $1 \mathrm{~cm}$ of the overlapping area as indicated in Figure 3. The resistance value is taken as a base to calculate the current distribution along the contact area. Each recorded value is the average of at least three reading on the same point.

\subsection{Experimental procedure}

A high precision 4-wire micro-ohmmeter with a maximum resolution of $0.02 \mu \Omega$ and fixed current 20 Ampere was used for all resistance measurements. It was essential part of all joints to clean all used bus- bar carefully by steel brush.

1. The material resistance was measured at $1 \mathrm{~cm}$ of the copper bus- bar it is found to be $0.44 \mu \Omega$, it is exactly as the calculated value for $1 \mathrm{~cm}$, 40x10 $\mathrm{mm}^{2}$ copper bus- bar

2. The overlapping distance was divided into several point with distance $1 \mathrm{~cm}$, the material resistance was measured using the same micro ohm.

3. The measured values are taken as base to calculate the current distribution along the contact area.
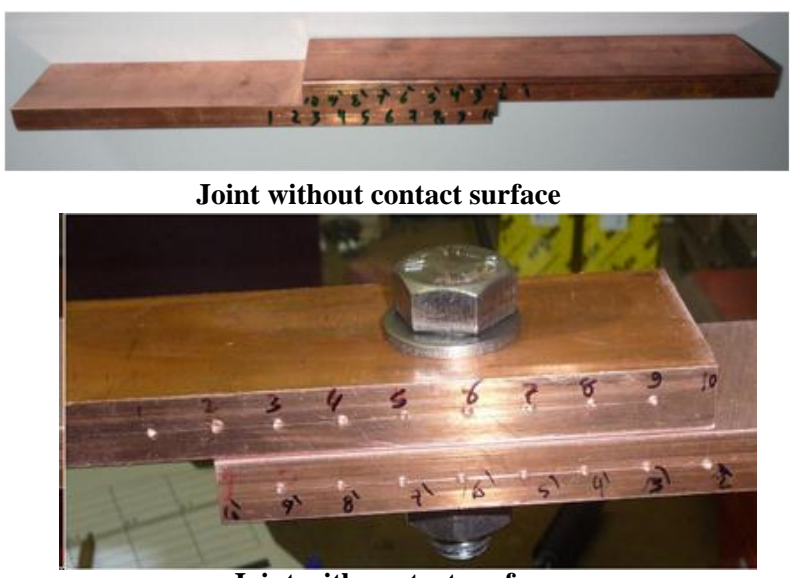

Joint with contact surface

Figure 2: photograph for the copper joint indicated measuring poits.

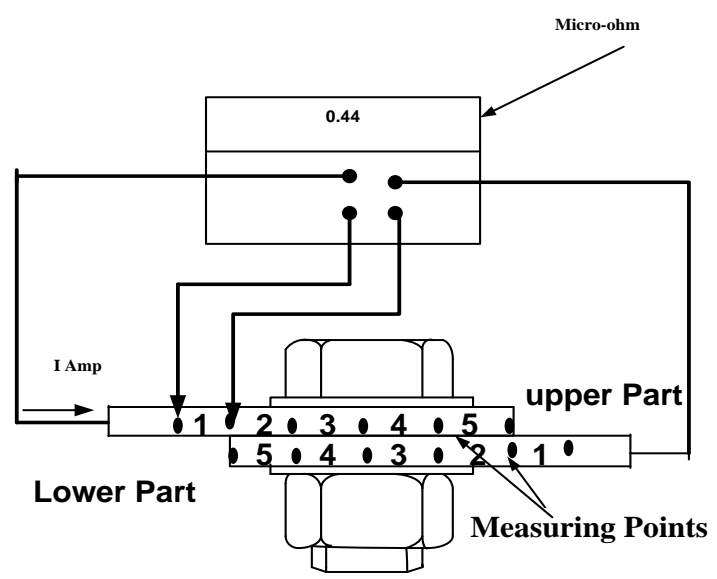

Figure 3 Schematic diagram of Joint Measurement Circuit (overlap $40 \mathrm{~mm}$ ).

\subsection{Experimental results}

\section{a. Material resistance}

Figure $4 \mathrm{a}$ and $\mathrm{b}$ show the measured material resistance over the contact area of $40 \mathrm{~mm}$ and $80 \mathrm{~mm}$ overlapping distance respectively, it is clear from the figure that for both ideal and normal joint, the measured material resistance decrease when measurement have been done closer to the end of the busbar. Furthermore, for both contact parts the material resistances of the end parts are very small and the same observation can be noticed in the case of Ideal joint.

Figure 5 gives the experimental results of the material resistance over the contact area at varying tightening torque for $40 \times 10 \mathrm{~mm}^{2}$ copper bus-bars of $40 \mathrm{~mm}$ overlapping distance, from the figure it can deduce that although increasing torque leads to increase the contact area, it have no effect on the measured material resistance.

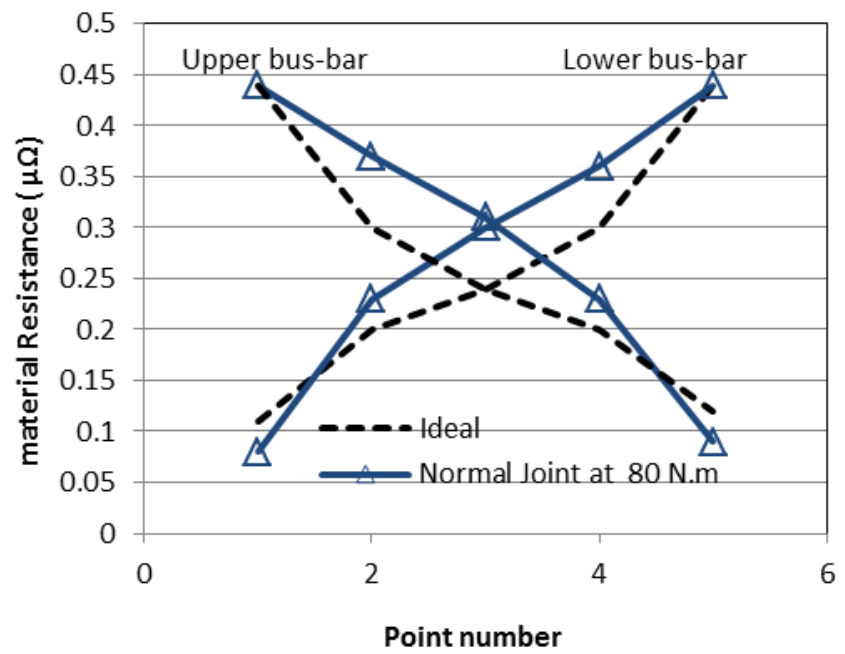

a. Overlap $40 \mathrm{~mm}$ 


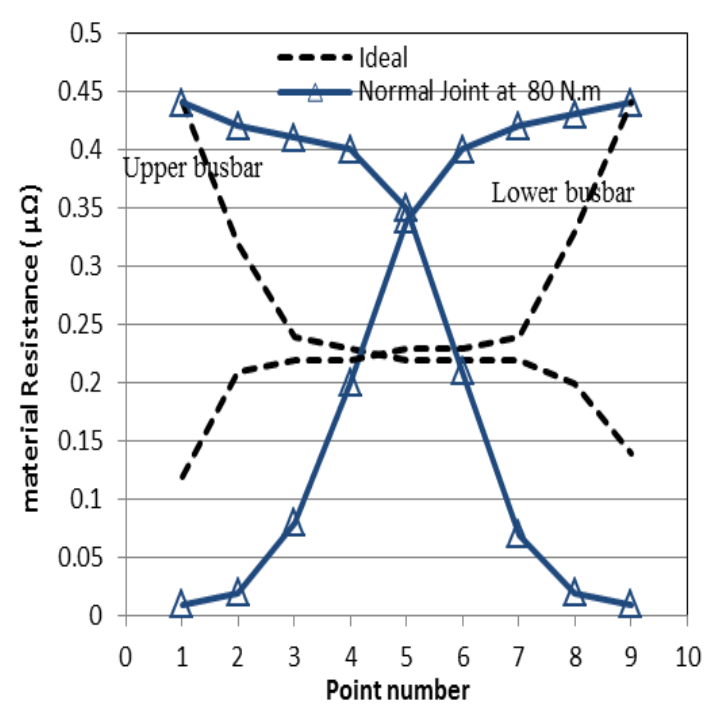

b. Overlap $80 \mathrm{~mm}$

Figure 4: Material resistance along contact area of Ideal joint and normal Joint at 80 N.m torque.

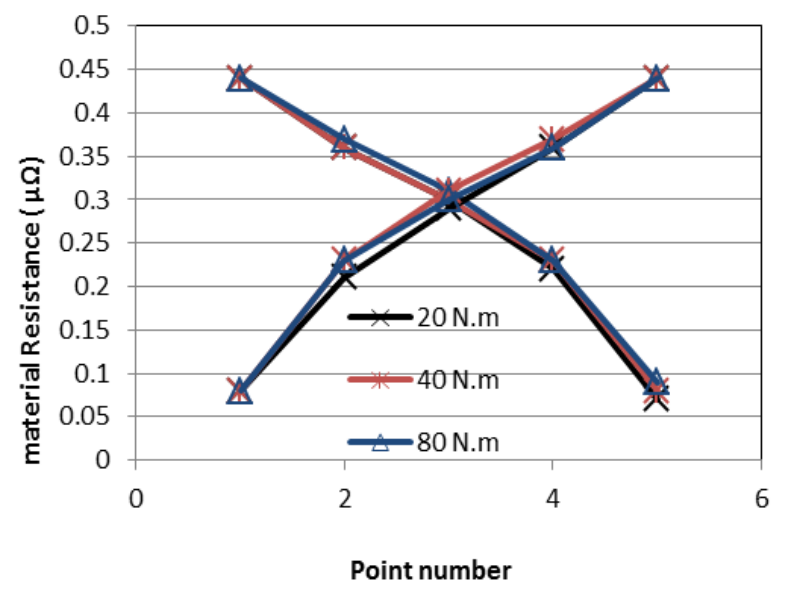

Figure 5: Material resistance along contact area at different torque

\section{b. current distribution}

The material resistance should not change along the busbar, the change in measuring material resistance means changing in the voltage drop along the corresponding distance which is a results of changing current pass in this part of the bus-bar, hence The joint resistance as recorded by the micro- ohmmeter is based on the current flowing through the joint, so the measured material resistance can be taken as a reference to calculate the percentage distribution along the overlapping distance, the following equation can be used to obtain the percentage current on $1 \mathrm{~cm}$ from the overlapping distance :

$\mathrm{I} \%=\mathrm{Rm}$ (measured) $* \mathrm{It} / \mathrm{Rm}$ (Exact)

Eq. 7 Where:
$\mathrm{Rm}$ (measured): is the recorded value from microohmmeter

It: total current passed from micro ohm

$\mathrm{Rm}$ (Exact): exact material resistance for $1 \mathrm{~cm}$ from copper bus-bar

Figure 6 a and $\mathrm{b}$ show the calculated current, as a percentage from the total current, over the contact area of $40 \mathrm{~mm}$ and $80 \mathrm{~mm}$ overlapping distance respectively.

From Figure 6 one can deduce that more than $85 \%$ of current pass before current reach $75 \%$ of over lapping distance, and only $2.5 \%$ of current pass from the last section of the over lapping area, the current pass from the bus-bar terminal reduce with increasing overlapping distance, but In the case of ideal joint the current are more uniform, and the last section curry about $30 \%$ of the total current as seen from Figure 6 . It is clear that the last section of bus-bar material for both contact parts are not included during measuring the initial joint resistance of bus-bar connection.

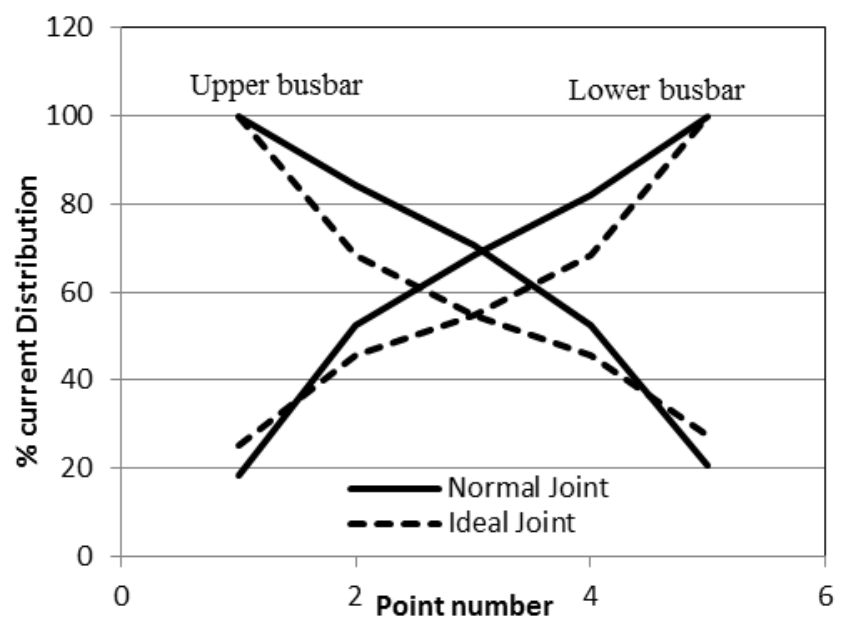

a. Overlap $40 \mathrm{~mm}$

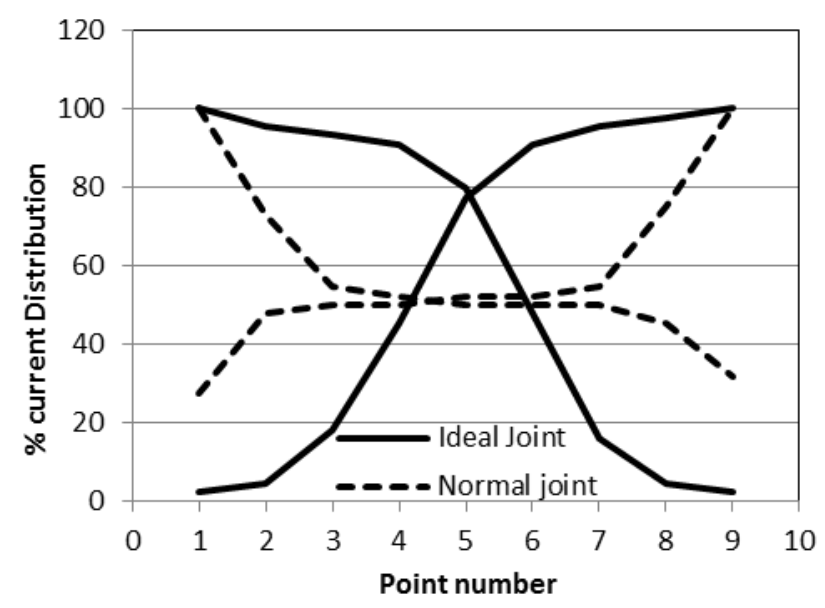

b. Overlap $80 \mathrm{~mm}$

Figure 6 : Current distribution over the contact area for ideal and normal joint ( a) over lap $40 \mathrm{~mm}$ (b) overlap $80 \mathrm{~mm}$ 


\section{Simulation Description}

\section{a. Geometry}

In Figure 7 we can see the 3-D-geometry of the model. In view of the axial symmetry of the system, a copper bus-bar was considering in calculation, two $40 \times 10 \mathrm{~mm}^{2}$ copper bus-bar, connected together, notice that there is a perfect electrical contact between the bodies, ideal connector, the overlap area is increased to calculate the overlap/ thickness ratio. SOLID69 Element was used in the simulation process; SOLID69 has a 3-D thermal and electrical conduction capability.

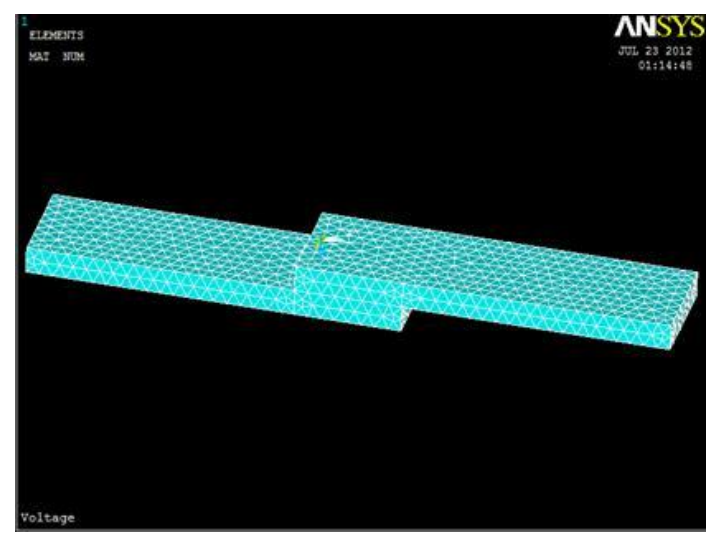

Figure 7: Finite element model of 40x10 copper busbar without hole

\section{b. Results and Validation}

Figure $8 \mathrm{a}$ and $\mathrm{b}$ show the calculation result for the copper bus-bar with and without hole respectively.

Figure 9, (a) shows the simulation result for ideal performance factor as a function of overlap/thickness ratio of the joint. The ideal performance factor decrease with the increase of overlap/thickness. The result shows that the optimal overlap length in a bolted joint should be not less than 4 times the bus-bar thickness Figure 9, (a); this result have a good agreement with that we have experimentally, furthermore this simulation result is consistent with the experimental measurement [1] Figure 9 (b).

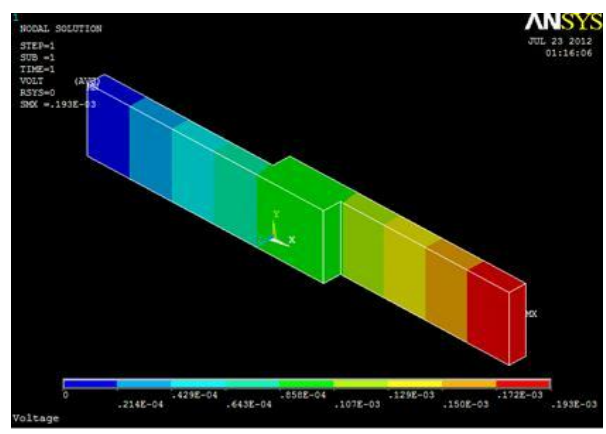

(a)

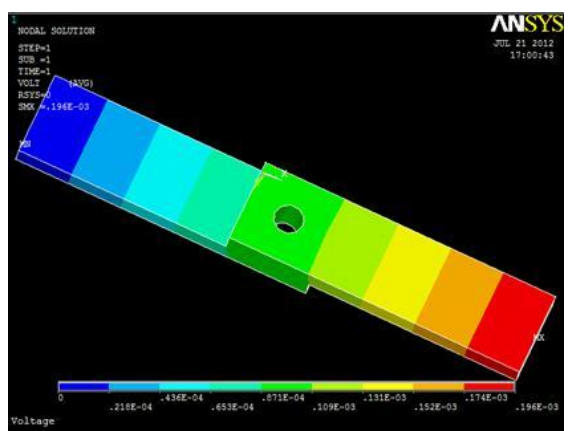

(b)

Figure 8: Calculation result of the $40 \times 10$ copper busbar a) without hole b) with hole $12 \mathrm{~mm}$ diameter

Figure 10 shows the simulation results for the resistance and performance factor as a function of hole diameter, from the figure it is clear that increasing hole diameter increasing the joint resistance and performance factor, this ascribed to decreasing material volume.

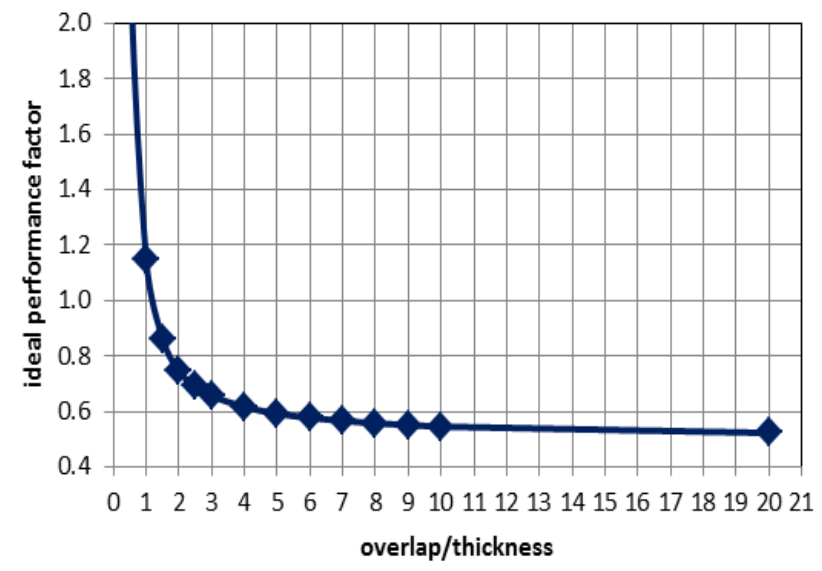

(a)

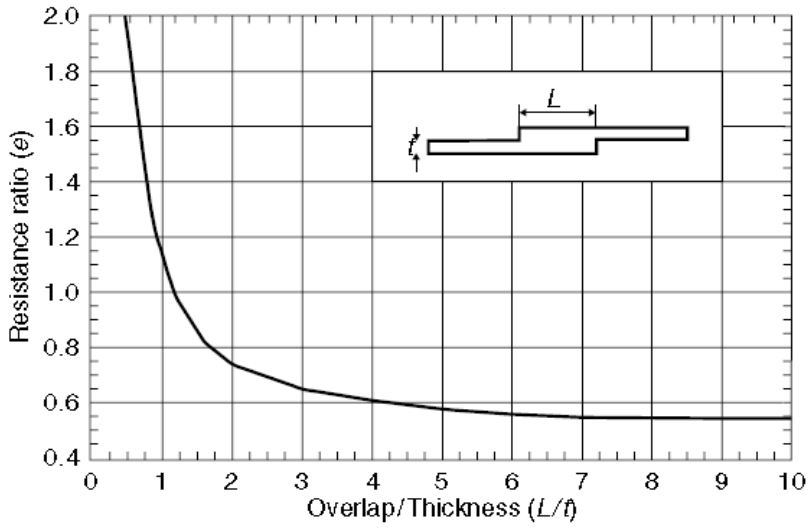

(b)

Figure 9: Ideal Performance factor as a function of overlap/thickness (a) calculate from the model (b) measured [1]. 


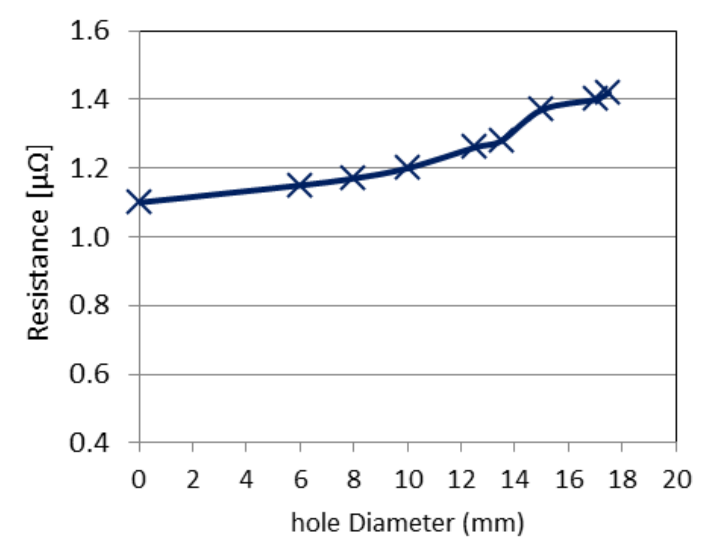

Figure 10: contact resistance as a function of hole diameter without contact surface (ideal connector

\section{Conclusions}

In this work, the factors effect on initial joint resistance in bolted power connection and the current distribution along aver lapping rejoin was studied experimentally The results may be summarized as follows:

1. The material resistance of the two ends of contact part has no effect on the joint resistance of bolted connections

2. Increasing of tightening torque has no effect on the current distribution along the contact area.

3. Increasing hole diameter has a negative impact in performance factor.

4. The simulation result showed that the overlap distance should be not less than 4 times the busbar thickness.

5. the results can contribute in finding suitable model or (lumped circuit) for bus-bar joint

\section{References}

[1] M. Braunovic', V. Konchits and N. Myshkin, , CRC Press Taylor Francis Group, 2007.

[2] R. Holm, Electrical Contacts - Theory and Applications,, 4th Ed., Springer-Verlag, Berlin 1967.

[3] L. Bily OVE, C. Hildmann, G. Moustafa, S. Grossmann, R. Kleveborn and L. Charlshem, "Experimental investigations to the joint resistance of bolted substation and transmission line connectors and its conformity to test standards," cigre 2010,August 22 to 27, 2010, pp. 358-361.

[4] Ghareeb Moustafa, Steffen Grossmann , Mazen Abdel-Salam, S. S. Dessouky and Samir M. ElMakkawy, "Joint Resistance of Bolted Copper Bus-Bar Connections as influenced by Mechanical Contact Devices Material and Configuration," MEPCON'09, 13th International Middle East Power Systems Conference December 20-23, 2009, Assiut University, Assiut, Egypt.

[5] Michael T. Singer and Kristopher Kshonze, "Electrical Resistance Of Random Rough Contacting Surfaces Using Fractal Surface Modeling", Proceedings of the Thirty-Seventh IEEE Holm Conference on Electrical Contacts, 6-9 Oct 1991,PP. 73-82..

[6] Ralf Bergmann, Helmut Löble, Helmut Böhme and Steffen Grossmann, "Model To Assess The Reliability of Electrical Joints", 18th international Conference on Electrical Contacts, chicago 16-20.9.19996, PP.173-179..

[7] Steffen Grossmann, Helmut Löbl and Helmut Böhme, "Contact Lifetime of Connections in Electrical Power System", 16th International Conference on Electrical Contacts ,Loughborough University of Technology, 1992.

[8] Sebastian Dreier, Steffen Großmann , Ghareeb Moustafa, Diego Guimaraes dos Santos, Brigida Ramati Pereira da Rocha and Valquiria Gusmão Macedo "Long-term Behavior of Static Electrical Connections in Areas with Harsh Environmental Conditions" Cigre Since and Engineering, Volume $\mathrm{N}^{\circ} 3$, October 2015, PP: 40-52.

[9] M. Braunovic, "Aluminum Connections: Legacies Of The Past," Proceedings of the Fortieth IEEE Holm Conference on Electrical Contacts, pp. 1- 31, 17-19 Oct. 1994. 